05,11

\title{
Магнитные фазы и неоднородные микромагнитные структуры в феррит-гранатовой пленке с ориентацией (210)
}

\author{
(C) Р.М. Вахитов, Р.P. Исхакова, А.Р. Юмагузин \\ Физико-технический институт, Башкирский государственный университет, \\ Уфра, Россия \\ E-mail: VakhitovRM@Yahoo.com
}

(Поступила в Редакцию 15 сентября 2017 г.)

Теоретически исследуются возможные магнитные состояния кубического ферромагнетика с наведенной вдоль направления [210] одноосной анизотропией. Показано, что ориентационная фазовая диаграмма магнетика является нетривиальной и допускает существование трех типов магнитных фаз, различающихся трансформационными свойствами, а также наличие пятерных точек, изоструктурных фазовых переходов и т.д. Установлено, что магнитные неоднородности независимо от значений параметров материала имеют общую структуру - они соответствуют 180-градусным доменным границам с некруговой траекторией вектора намагниченности. Найденные особенности однородных и неоднородных магнитных состояний в данных материалах позволяют объяснить характер проявления в них флексомагнитоэлектрического эффекта.

Работа выполнена при поддержке РФФИ (грант № 16-02-00336).

DOI: 10.21883/FTT.2018.05.45788.327

\section{1. Введение}

Синтезированные около 30 лет назад ферритгранатовые пленки с ориентацией (210) зарекомендовали себя в процессе их изучения как перспективные многофункциональные материалы, которые могут быть использованы и в устройствах магнитной записи [1], и в высокочувствительных датчиках для визуализации малых неоднородных полей [2] и т.д. В то же время после обнаружения в этих пленках гигантского магнитоэлектрического (МЭ) эффекта при комнатных температурах [3] о них заговорили как о магнитоэлектрических материалах. Кроме того, в последующих исследованиях в них был открыт новый сильный МЭ эффект, который заключался в смещении доменных границ (ДГ) под действием внешнего электрического поля [4]. Интересной особенностью наблюдаемого эффекта явилось его зависимость от ориентации пленки: в пленках ферритовгранатов с ориентацией (210) МЭ эффект проявлялся наиболее сильно, в пленках с ориентацией (011) - более слабо, а в пленках с ориентацией (111) МЭ эффект вообще не наблюдался. В литературе было предложено два возможных объяснения наблюдаемого явления. Первое - это МЭ эффект, который обусловлен наличием неоднородного магнитоэлектрического взаимодействия в рассматриваемых материалах [4]. Согласно [5], данный механизм проявляется лишь в том случае, когда существующие в них ДГ имеют неблоховскую структуру. Второе - эффект определяется условиями проведения эксперимента и особенностями ориентационной фазовой диаграммы (ОФД) изучаемой пленки [6]. Таким образом, из сказанного следует, что для объяснения результатов [4] необходимо провести анализ однородных и неоднородных магнитных состояний, возможных в пленке типа (210) ферритов-гранатов, т.к. подобные исследования в них до сих пор не проводились (за исключением работы [6], где приведен анализ ОФД пленки типа (210) в пренебрежении вкладом кубической анизотропии).

\section{2. Однородные магнитные состояния пластины (210) с комбинированной анизотропией}

Вначале изучим равновесные направления вектора намагниченности М в кубическом ферромагнетике с наведенной вдоль оси [210] одноосной анизотропией. Будем считать, что образец представляет собой однородно намагниченную пластину конечной толщины. Систему координат выбираем так, что ось $O z$ перпендикулярна плоскости пластины и совпадает с направлением [210], ось $O x$ параллельна направлению [120], а $O y-$ направлению [001]. Тогда плотность энергии однородных состояний магнетика $\varepsilon_{m a}$, включающая плотности энергий наведенной одноосной и кубической анизотропий, a также плотность энергии размагничивающих полей пластины, запишется в виде

$$
\begin{aligned}
\varepsilon_{m a} & =K_{u} \sin ^{2} \theta+K_{p} \sin ^{2} \theta \cos ^{2} \varphi-\frac{3}{2} K_{p} \sin \theta \cos \theta \cos \varphi \\
& +\frac{K_{1}}{25}\left(25 \sin ^{4} \theta \sin ^{2} \varphi \cos ^{2} \varphi+25 \sin ^{2} \theta \cos ^{2} \theta \sin ^{2} \varphi\right. \\
& +4 \sin ^{4} \theta \cos ^{4} \varphi+4 \cos ^{4} \theta+\sin ^{2} \theta \cos ^{2} \theta \cos ^{2} \varphi \\
& \left.+12 \sin \theta \cos ^{3} \theta \cos \varphi-12 \sin ^{3} \theta \cos \theta \cos ^{3} \varphi\right),
\end{aligned}
$$

где $\theta$ и $\varphi-$ полярный и азимутальный углы вектора $\mathbf{M}$, $K_{u}, K_{p}-$ константы, соответственно перпендикуляр- 
ной и ромбической компонент наведенной одноосной анизотропии [6], $K_{1}$ - первая константа кубической анизотропии. Здесь учитывается, что в величину константы $K_{u}$ вносят вклад и размагничивающие поля пластины: $K_{u}=K_{u}^{\prime}-2 \pi M_{s}^{2}$, где $M_{s}-$ намагниченность насыщения.

Однородные магнитные состояния рассматриваемого магнетика находятся из минимума (1), т. е. из следующих уравнений:

$$
\frac{\partial \varepsilon}{\partial \theta}=0, \quad \frac{\partial \varepsilon}{\partial \varphi}=0
$$

при выполнении условий

$$
\frac{\partial^{2} \varepsilon}{\partial \theta^{2}}>0, \quad \frac{\partial^{2} \varepsilon}{\partial \varphi^{2}}>0, \quad \frac{\partial^{2} \varepsilon}{\partial \theta^{2}} \frac{\partial^{2} \varepsilon}{\partial \varphi^{2}}-\left(\frac{\partial^{2} \varepsilon}{\partial \theta \partial \varphi}\right)^{2}>0
$$

Из анализа полученных соотношений для случая $K_{1}=0$ следует, что в рассматриваемом магнетике возможно существование трех типов магнитных фаз; их обозначения, ориентации и области существования имеют вид [7]:

1. $\theta=\pi / 2, \varphi=\pi / 2,3 \pi / 2 ; \mathbf{M} \|[001],[00 \overline{1}]$ - симметричная фаза $P_{[001]}$. Область ее существования определяется соотношениями: $K_{p}>0, K_{u}<-\frac{9}{16} K_{p}$.

2. $\theta=\theta_{0}, \varphi=0$ и $\theta=\pi-\theta_{0}, \varphi=\pi ; \mathbf{M} \|[u v 0]-$ угловая фаза $P_{[u v 0]}$. Область ее устойчивости $K_{u}<0 \cup K_{p}>0, K_{u}>-\frac{9}{16} K_{p}$. Здесь

$\theta_{0}=\operatorname{arctg}\left\{\left[\sqrt{4\left(K_{u}+K_{p}\right)^{2}+9 K_{p}^{2}}-2\left(K_{u}+K_{p}\right)\right] / 3 K_{p}\right\}$.

3. Третье возможное состояние магнетика представляет собой фазу типа „наклонная легкая плоскость“, которая задается соотношением

$$
\operatorname{tg} \theta=\frac{3}{4} \cos \varphi
$$

a ее ориентация определяется нормалью $\mathbf{n}=$ $=(0,4 / 5,-3 / 5)$. Существование этой фазы возможно на линии $K_{u}=-\frac{9}{16} K_{p}$. Выполнение последнего соотношения (вполне достижимого для пленок ферритовгранатов $[2,8])$, дает возможность зафиксировать с помощью (210)-ориентированной пленки сколь угодно малые поля, действующие на исходный образец. Данное свойство таких пленок может найти применение в высокочувствительных датчиках малых неоднородных полей.

Между фазами $P_{[001]}$ и $P_{[u v 0]}$ на линии $K_{p}=0, K_{u}<0$ имеет место спин-переориентационный фазовый переход (СПФП) второго рода: переход вектора М между данными состояниями происходит путем непрерывной спиновой переориентации. В то же время на линии $K_{u}=-\frac{9}{16} K_{p}\left(K_{p}>0\right)$. Переход между фазами $P_{[001]}$ и $P_{[u v 0]}$ также происходит непрерывно, но посредством двух СПФП второго рода через промежуточное состояние типа „легкая наклонная плоскость“. Это связано с тем, что вектор М в обеих фазах при достижении линии
СПФП $\left(K_{u}=-\frac{9}{16} K_{p}\right)$ лежит в плоскости, определяемой соотношением (4).

Следует отметить отсутствие в перечне найденных фаз симметричной фазы $P_{[210]}(\theta=0, \pi ; \mathbf{M} \|[210])$, что связано с наличием в пленке с ориентацией (210) „скошенной ромбической анизотропии“ [2], описываемой третьим слагаемым в выражении (1). Кроме того, необходимо подчеркнуть, что полученные результаты согласуются с аналогичными исследованиями равновесных направлений вектора намагниченности М, проведенными в $[6,9]$ для случая $K_{1}=0$.

Теперь рассмотрим влияние кубической анизотропии на однородные магнитные состояния (210)ориентированной пленки. В этом случае $\left(K_{1} \neq 0\right)$ ОФД рассматриваемого магнетика в переменных $\left(\mathfrak{x}_{1}, \mathfrak{x}_{2}\right)$, где $æ_{1}=K_{1} /\left|K_{u}\right|, æ_{2}=K_{p} /\left|K_{u}\right|$, значительно усложняется (см. рис. $1, a, b)$ : на ней наряду с симметричной $\left(P_{[001]}\right)$ и угловой $\left(P_{[u v 0]}\right)$ фазами появляются новые, в частности, фаза общего вида $P_{[u v w]}(\mathbf{M} \|[u v w])$. Следует отметить, что эти фазы отличаются друг от друга трансформационными свойствами: при изменении параметров материала (что может быть достигнуто действием внешних напряжений [10], либо термическим воздействием и т.д. [7]) вектор намагниченности М в симметричной фазе, направленный вдоль одной из осей симметрии куба, не изменяет своей ориентации, в угловой фазе вектор М изменяет свою ориентацию, оставаясь лежать в одной из плоскостей симметрии куба, в фазе общего вида вектор М меняет свое направление так, что годограф вектора М описывает на сфере радиуса $M_{s}$ некруговую траекторию.

Симметричная фаза $P_{[001]}$ в отсутствие кубической анизотропии не возникает при $K_{u}>0$, т.к. одноосная анизотропия типа „легкая ось“ препятствует возникновению любых равновесных направлений вектора М, лежащих в плоскости пластины. Однако при $K_{1} \neq 0$ возникает возможность того, что при $K_{u}>0$ фаза $P_{[001]}$ может стать устойчивой: условия для ее существования появляются при бо́льших величинах æ⿸ $\left(æ_{1}>20.14 ;\right.$ рис. $\left.1, a\right)$. Этому благоприятствует тот факт, что при $K_{1}>0$ легкими осями кубической анизотропии являются оси типа $\langle 100\rangle$.

Другой особенностью ОФД исследуемого магнетика при $K_{1} \neq 0$ является возникновение множества угловых фаз типа $P_{[u v 0]}$ как при $K_{u}>0$ (рис. $\left.1, a, b\right)$, так и при $K_{u}<0$ (рис. 2). Они относятся к одной группе симметрии и отличаются лишь значениями угла $\theta$. Между ними возможны СПФП первого рода, которые называют изоструктурными СПФП $[11,12]$. Линии таких переходов, как правило, заканчиваются в критических точках $C_{i}(i=1,2, \ldots)$, что имеет аналогию с термодинамической системой ,жидкость-пар“, для которой кривая фазового равновесия при повышении температуры также заканчивается критической точкой: при температуре выше критической исчезают всякие различия между исходными фазами [13]. В частности, при $K_{u}>0$ 

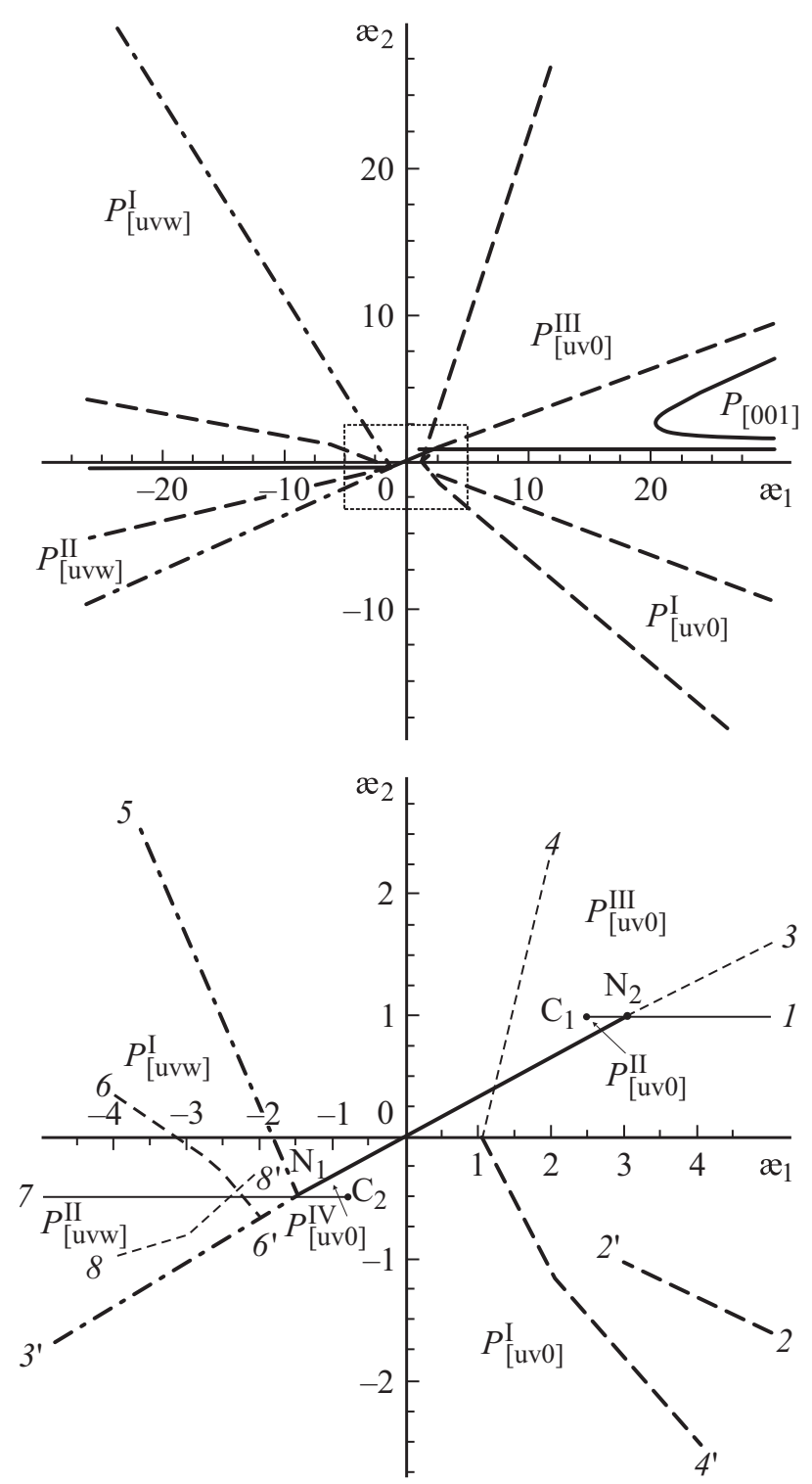

Рис. 1. ОФД (210)-ориентированной пластины ферритовгранатов для значений $K_{u}>0$. Здесь ОФД, соответствующая случаю $(b)$, является увеличенной копией выделенного (пунктирной линией) участка на ОФД $(a)$. Сплошные линии соответствуют линиям СПФП первого рода, штрихпунктирные - линиям СПФП второго рода, штриховые - границам устойчивости магнитных фаз.

(pис. $1, a, b)$ на участке $C_{1} N_{2}$ линии $1-C_{1} \quad\left(æ_{2}=1\right)$ происходит изоструктурный СПФП первого рода между фазами $P_{[u v 0]}^{\mathrm{II}}$ и $P_{[u v 0]}^{\mathrm{III}}$. Здесь $N_{2}=(3 ; 1)$ - тройная точка, где сходятся границы областей существования трех фаз $P_{[u v 0]}^{\mathrm{I}}, P_{[u v 0]}^{\mathrm{II}}$ и $P_{[u v 0]}^{\mathrm{III}}$, а $C_{1}=(2.5 ; 1)-$ критическая точка. Точно такая же ситуация имеет место на участке $N_{1} C_{2}$ прямой 7-C $C_{2}\left(æ_{2}=-0.48\right)$, который также является линией изоструктурного СПФП первого рода между двумя угловыми фазами $P_{[u v 0]}^{\mathrm{I}}$ и $P_{[u v 0]}^{\mathrm{IV}}$. В данном случае $N_{1}=(-1.48 ;-0.48)$ - пятерная точка, где сходятся границы областей существования пяти фаз: $P_{[u v 0]}^{\mathrm{I}}, P_{[u v 0]}^{\mathrm{IV}}$,
$P_{[u v 0]}^{\mathrm{III}}, P_{u v w}^{\mathrm{I}}$ и $P_{[u v w]}^{\mathrm{II}}, C_{2}=(-0.87 ;-0.48)-$ критическая точка. Наличие пятерной точки на ОФД пленки (210) находится в соответствии с правилом фаз Гиббса для исследуемой системы [14]. Кроме того, при $K_{u}>0$ на ОФД (рис. $1, a, b)$ имеются также две фазы общего вида $\left(P_{[u v w]}^{\mathrm{I}}\right.$ и $\left.P_{[u v w]}^{\mathrm{II}}\right)$, которые разделены между собой линией $7-N_{1}\left(\mathfrak{x}_{2}=-0.48\right)$, являющийся линией СПФП первого рода между ними.

Следует отметить, что при $æ_{1} \leq 2.5$ полностью исчезают различия между угловыми фазами $P_{[u v 0]}^{\mathrm{II}}$ и $P_{[u v 0]}^{\mathrm{III}}$. Поэтому закритическую область $\left(\mathfrak{x}_{1}<2.5\right)$, прилегающую к участку $N_{1} N_{2}$ линии $3-3^{\prime}$, но расположенную выше нее, можно считать областью фазы $P_{[u v 0]}^{\mathrm{II}}$. Аналогичная ситуация имеет место и в закритической области $\left(\mathfrak{x}_{2}>-0,48\right)$ изоструктурного СПФП первого рода между фазами $P_{[u v 0]}^{\mathrm{I}}$ и $P_{[u v 0]}^{\mathrm{IV}}$; здесь переход между ними происходит непрерывно. А область, расположенную вблизи, но ниже участка $N_{1} N_{2}$, можно считать областью существования фазы $P_{[u v 0]}^{\mathrm{IV}}$. В этом случае участок $N_{1} N_{2}$ будет являться линией СПФП первого рода между фазами $P_{[u v 0]}^{\mathrm{II}}$ и $P_{[u v 0]}^{\mathrm{IV}}$. Соответственно на линии 5-N $N_{1}$ имеет место СПФП второго рода между фазами $P_{[u v w]}^{\mathrm{I}}$ и $P_{[u v 0]}^{\mathrm{IV}}$, а на линии $3^{\prime}-N_{1}-$ СПФП второго рода между $P_{[u v w]}^{\mathrm{II}}$ и $P_{[u v 0]}^{\mathrm{I}}$.

При $K_{u}<0$ ОФД исследуемой пленки не меняется существенным образом (рис. 2). В этом случае симметричная фаза $P_{[001]}$ в основном устойчива при $æ_{1}>0$, как и должно быть, однако к сказанному выше насчет условий устойчивости данной фазы в области $\mathfrak{x}_{1}>0$ добавляется еще один фактор, благоприятствующий фазе $P_{[001]}$ : при $K_{u}<0$ одноосная анизотропия становится легкоплоскостной. Данный фактор позволяет существовать симметричной фазе $P_{[001]}$ и при некоторых отрицательных значениях $æ_{1}$. Если термодинамический

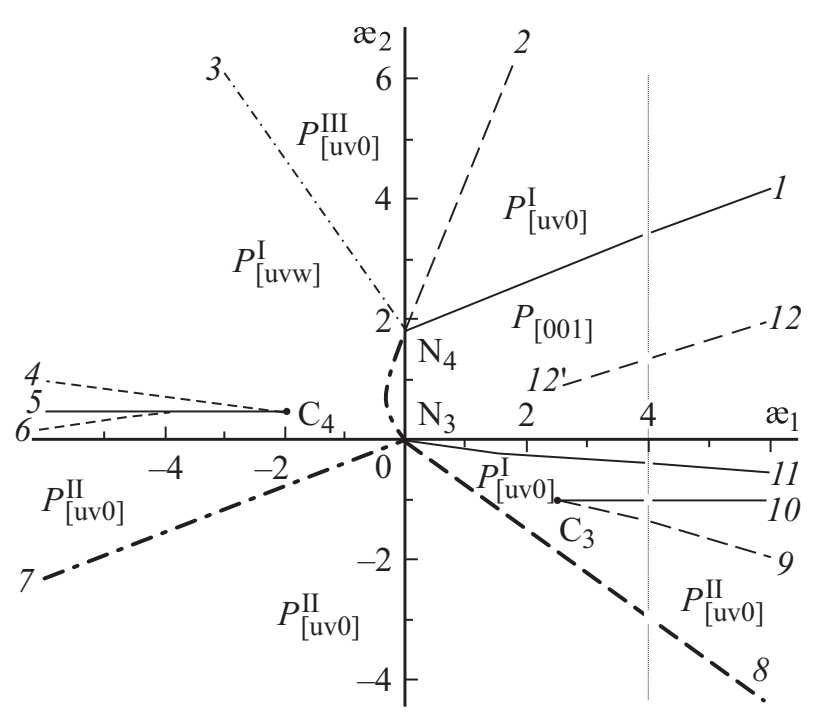

Рис. 2. ОФД пластины $(210)$ для значений $K_{u}<0$. Обозначения кривых те же, что и на рис. $1(a, b)$. 
путь, вдоль которого можно отслеживать переход системы из одного состояния в другое, выбрать совпадающим с линией $æ_{1}=a(a>2.5)$ то, двигаясь вдоль нее в сторону возрастающих значений æ 2 , рассматриваемая магнитная система будет переходить из фазы $P_{[u v 0]}^{\mathrm{II}}$ В фазу $P_{[u v 0]}^{\mathrm{I}}$, потом из фазы $P_{[u v 0]}^{\mathrm{I}}$ в фазу $P_{[001]}$, а затем из фазы $P_{[001]}$ в фазу $P_{[u v 0]}^{\mathrm{III}}$ (рис. 2). При этом линии $10-C_{3}, 11-N_{3}$ и $1-N_{4}$ будут являться линиями СПФП первого рода, соответственно между фазами $P_{[u v 0]}^{\mathrm{II}}$ и $P_{[u v 0]}^{\mathrm{I}}, P_{[u v 0]}^{\mathrm{I}}$ и $P_{[001]}$, а также $P_{[001]}$ и $P_{[u v 0]}^{\mathrm{III}}$. Подобная ситуация имела место при $K_{u}>0$, однако при $K_{u}<0$ и $\mathfrak{x}_{1}<0$ симметричная фаза $P_{[001]}$ на участке $N_{3} N_{4}$ переходит в фазу общего вида посредством СПФП второго рода, что не имеет аналогии в случае $K_{u}>0$. Здесь $N_{3}=(0 ; 0)$ и $N_{4}=(0 ; 1.78)$ - тройные точки, где сходятся соответственно фазы $P_{[001]}, P_{[u v 0]}^{\mathrm{I}}$ и $P_{[u v 0]}^{\mathrm{III}}$, а также $P_{[001]}, P_{[u v 0]}^{\mathrm{I}}$ и $P_{[u v 0]}^{\mathrm{II}}$.

В области, лежащей между прямыми $3-N_{4}, 7-N_{3}$ и отрезком $N_{3} N_{4}$, которые являются линиями СПФП второго рода, устойчива фаза общего вида. При этом на линиях $3-N_{4}$ и $7-N_{3}$ имеет место непрерывные переходы между фазами соответственно, $P_{[u v 0]}^{\mathrm{III}}$ и $P_{[u v w]}^{\mathrm{I}}$, $P_{[u v 0]}^{\mathrm{II}}$ и $P_{[u v w]}^{\mathrm{II}}$, а на участке $N_{3} N_{4}-$ между фазами $P_{[u v w]}^{\mathrm{I}}$ и $P_{[001]}$. При $\mathfrak{x}_{1}<-2$ область существования фазы $P_{[u v w]}^{\mathrm{I}}$ превращается в две области, разделенные прямой $5-C_{4}$. Последняя является линией изоструктурного СПФП первого рода между фазами $P_{[u v w]}^{\mathrm{I}}$ и $P_{[u v w]}^{\mathrm{II}}$, которые и существуют в вышеупомянутых областях. Эти фазы представляют собой (по симметрии) фазы общего вида и различаются лишь значениями углов $\theta$ и $\varphi$. В закритической области (определяемой неравенством $\mathfrak{x}_{1}>-2$ и расположенная справа от критической точки $\left.C_{4}=(-2 ; 0.47)\right)$, переход между ними происходит непрерывно, т.е. они фактически сливаются в одну фазу - фазу общего вида $(\mathbf{M} \|[u v w])$. Аналогичная ситуация имеется и в области, заключенной между линиями $7-N_{3}$ и $11-N_{3}\left(æ_{2}<0\right)$, где устойчива угловая фаза $(\mathbf{M} \|[u v 0])$. Эта область при $\mathfrak{x}_{1}>2.5$ также переходит в две подобласти, разделенные прямой $10-C_{3}$ $\left(\mathfrak{x}_{2}<-1\right)$, являющейся линией изоструктурного СПФП первого рода между фазами $P_{[u v 0]}^{\mathrm{I}}$ и ${ }_{[u v 0]}^{\mathrm{II}}$. В данном случае в области, расположенной выше линии $10-C_{3}$, $\left(\mathfrak{x}_{2}<-1\right)$ устойчива фаза $P_{[u v 0]}^{\mathrm{I}}$, а ниже вышеуказанной линии $\left(\mathfrak{x}_{2}<-1\right)-$ фаза $P_{[u v 0]}^{\mathrm{II}}$, а точка $C_{3}=(2.5 ;-1)$ соответственно является критической точкой изоструктурного СПФП первого рода между этими фазами. В закритической области $\left(\mathfrak{x}_{2}<2.5\right)$ переход между ними происходит непрерывно, т.к. различие между фазами $P_{[u v 0]}^{\mathrm{I}}$ и $P_{[u v 0]}^{\mathrm{II}}$ исчезает.

Следует отметить одну характерную особенность ОФД рассматриваемого магнетика, имеющую место как при $K_{u}>0$, так и при $K_{u}<0$ : между симметричной $\left(P_{[001]}\right)$ и угловой $\left(P_{[u v 0]}\right)$ фазами возможны только СПФП первого рода, а между угловой и фазой общего вида - СПФП как первого, так и второго рода. Это объясняется тем, что вектор М в угловой фазе при изменении параметров материала всегда лежит в плоскости, перпендикулярной оси [001]. Поэтому возможен только скачкообразный переход $P_{[u v 0]} \leftrightarrows P_{[001]}$.

Как видно из приведенного анализа, топология ОФД (210)-ориентированной пластины является достаточно сложной: на ней имеется множество угловых фаз, которые могут перейти друг в друга посредством СПФП первого рода. Соответствующие линии перехода обрываются в критической точке. Такая же ситуация имеет место и для фаз общего вида (М $\|[u v w])$, между которыми также может произойти изоструктурный СПФП первого рода. Кроме того, на ОФД присутствуют тройные и пятерные точки и т.д.

Очевидно, такой характер ОФД пленки (210) обусловлен выбранным направлением индуцирования одноосной анизотропии (вдоль оси [210]), которое не совпадает ни с одной осью симметрии куба и приводит к разложению наведенной одноосной анизотропии на несколько составляющих. Соответственно равновесное направление вектора намагниченности М находится под конкурирующим влиянием сразу нескольких анизотропий различной симметрии: перпендикулярной, ромбической, „скошенной“ ромбической и кубической. В результате магнитная симметрия пленки является достаточно низкой; в ней имеется лишь один элемент - плоскость отражения $\sigma_{v}$, которая совпадает с плоскостью (001). Именно поэтому на ОФД магнетика имеется всего лишь одна симметричная фаза $P_{[001]}$, а в угловых фазах изменение ориентации вектора М происходит только в плоскости (001). Нетрудно предположить, что магнитные неоднородности, возможные в изучаемом магнетике, будут также иметь нетривиальную структуру.

\section{3. Возможные микромагнитные структуры}

При рассмотрении неоднородных состояний примем, что в системе координат, введенной ранее, ось $O y$ совпадает с направлением, вдоль которого магнетик неоднороден. Тогда энергию магнитных неоднородностей, взятую с учетом обменного взаимодействия $A$, энергии магнитной анизотропии $\varepsilon_{m a}$, магнитостатической энергии объемных зарядов, локализованной в ДГ $[15,16]$, и за вычетом энергии однородных состояний $\varepsilon_{0}$, запишем в виде

$$
\begin{aligned}
E= & \int\left\{A\left[\left(\frac{d \theta}{d y}\right)^{2}+\sin ^{2} \theta\left(\frac{d \varphi}{d y}\right)^{2}\right]+\varepsilon_{m a}+2 \pi M_{s}^{2}\right. \\
& \left.\times\left(\sin \theta \sin \varphi-\sin \theta_{m} \sin \varphi_{m}\right)^{2}-\varepsilon_{0}\right\} d y,
\end{aligned}
$$

где $\theta_{m}, \varphi_{m}-$ значения углов, определяющих вектор $\mathbf{M}$ $\left.\left(\theta_{m}=\theta(y \rightarrow \infty)\right), \quad \varphi_{m}=\varphi(y \rightarrow \infty)\right) \quad \varepsilon_{0}=\varepsilon_{m a}(y \rightarrow \infty)$. Здесь предполагается, что пластина является достаточно 


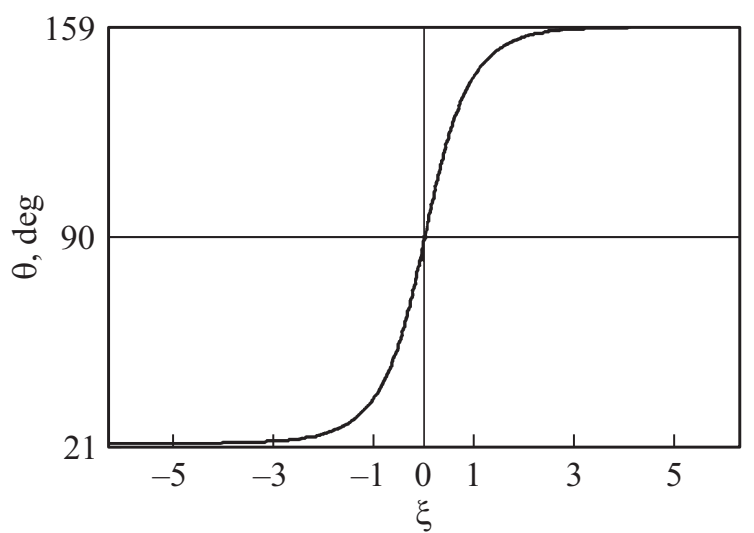

$a$
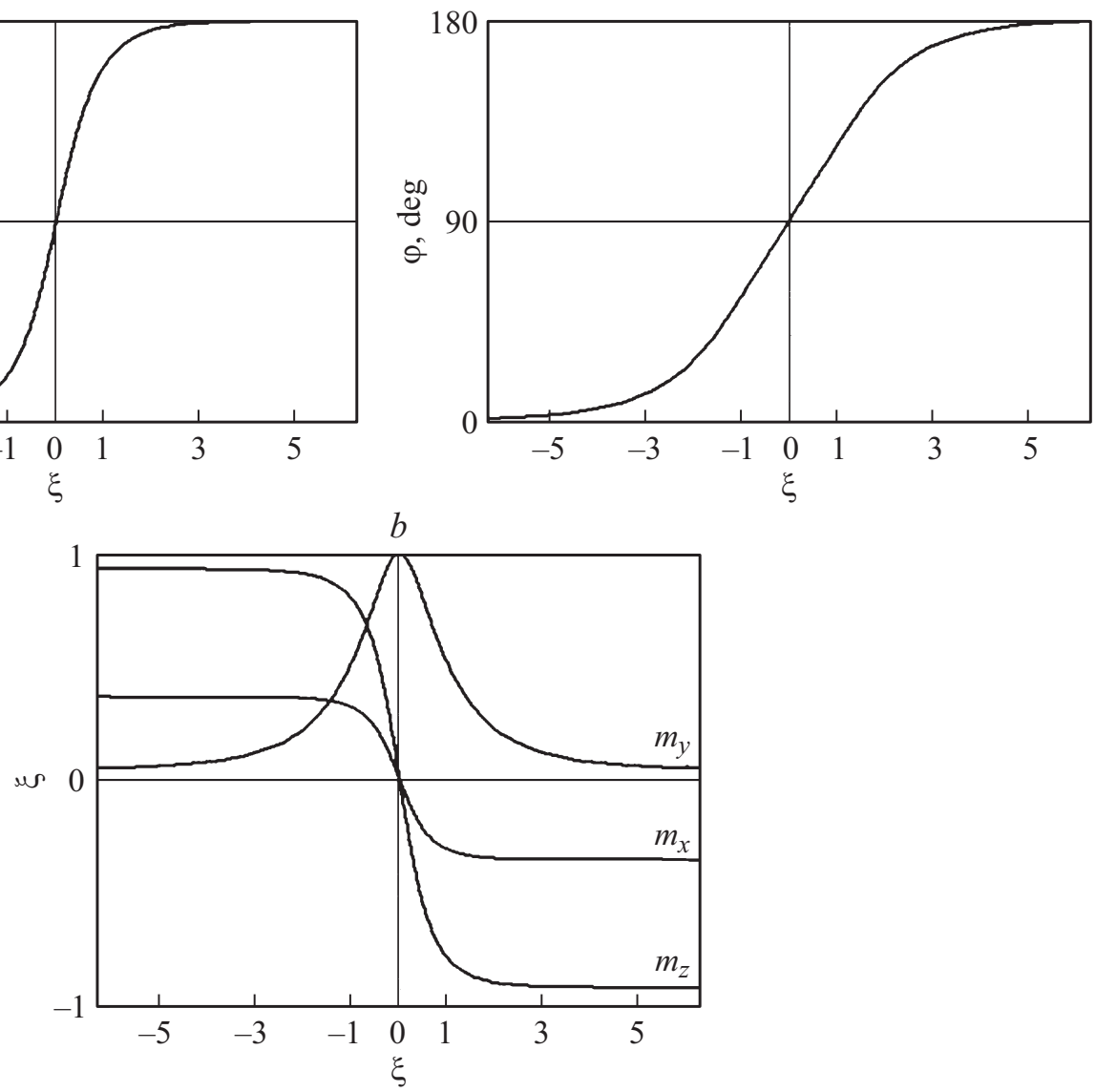

Рис. 3. Зависимости угловых переменных $\theta$ и $\varphi(a)$ единичного вектора $\mathbf{m}=\mathbf{M} / M_{s}$ и его компонент $m_{x}, m_{y}, m_{z}(b)$ от переменной $\xi$, иллюстрирующие распределение намагниченности в $180^{\circ}$-ДГ при следующих значениях материальных параметров: $\mathfrak{x}_{1}=-2, \mathfrak{x}_{2}=2, Q=5, K_{u}>0$.

толстой, и можно пренебречь влиянием размагничивающих полей, создаваемых поверхностными зарядами.

Уравнения Эйлера-Лагранжа, описывающие структуру магнитных неоднородностей, возможных в данном магнетике, после перехода к безразмерным величинам примут вид

$$
\begin{gathered}
\frac{d^{2} \theta}{d \xi^{2}}-\sin \theta \cos \theta\left(\frac{d \varphi}{d \xi}\right)^{2}=\frac{\partial f(\theta, \varphi)}{\partial \varphi}, \\
2 A \frac{d}{d \xi}\left(\sin ^{2} \theta \frac{d \varphi}{d \xi}\right)=\frac{\partial f(\theta, \varphi)}{\partial \varphi},
\end{gathered}
$$

где $\xi=y / \Delta_{0}, \Delta_{0}=\sqrt{A / K_{u}}-$ характерный размер магнитных неоднородностей $[15], f(\theta, \varphi)$ определяется выражением

$$
\begin{aligned}
f(\theta, \varphi)= & \left(\varepsilon_{m a}-\varepsilon_{0}\right) / 2 K_{u} \\
& +(2 Q)^{-1}\left(\sin \theta \sin \varphi-\sin \theta_{m} \sin \varphi_{m}\right)^{2}
\end{aligned}
$$

$Q=K_{u} / 2 \pi M_{s}^{2}-$ фактор качества материала.

Для нахождения решений уравнений (6) необходимо задать граничные условия, которые, исходя из симмет- рии магнетика, можно записать в виде

$$
\begin{gathered}
\theta(\xi \rightarrow-\infty)=\theta_{0}, \quad \theta(\xi \rightarrow+\infty)=\pi-\theta_{0}, \\
\varphi(\xi \rightarrow-\infty)=\varphi_{0}, \quad \varphi(\xi \rightarrow+\infty)=\varphi_{0}+\pi,
\end{gathered}
$$

где $\theta_{0}, \varphi_{0}$ - решения уравнений (2) при выполнении соотношений (3). Как видно из (8), они соответствуют 180-градусной ДГ (180-ДГ).

Очевидно, уравнения системы (6) представляют собой нелинейные дифференциальные уравнения второго порядка, которые из-за низкой симметрии исследуемого магнетика с энергией, описываемой выражением (5), могут быть решены только численными методами. В основу численной реализации нахождения решений системы (6) была положена методика, рассмотренная в работе [17]. Суть ее в следующем: вначале система (6) путем преобразования бесконечного интервала по координате $\xi$ к интервалу $[-1,1]$ по формуле $\eta=(2 / \pi) \operatorname{arctg} \xi$ с последующей заменой дифференциальных операторов конечными разностями (методом дискретизации) сводится к системе трансцендентных уравнений относительно искомых значений углов $\theta, \varphi$ в узлах сетки, а затем последняя решается численно с помощью итерационных вычислений, с использованием метода верхней 
$a$
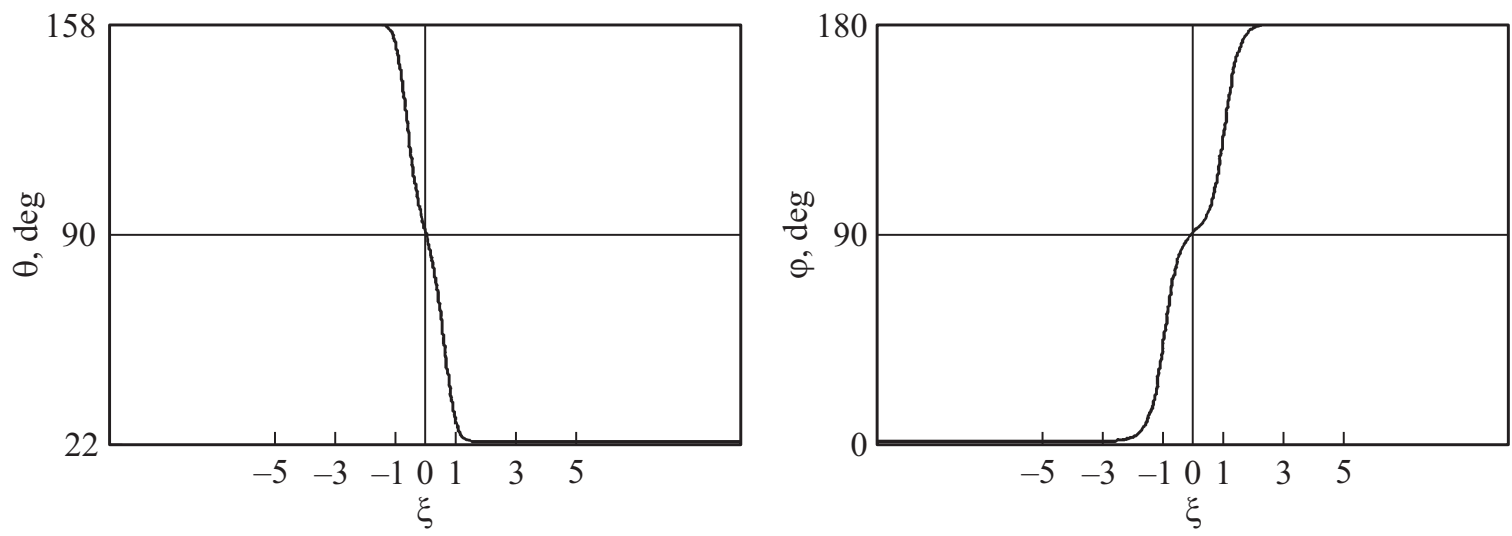

$b$

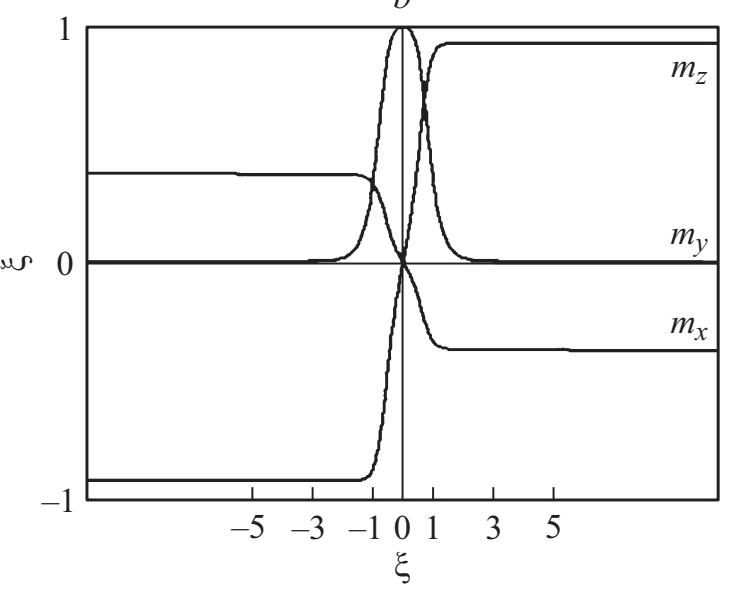

Рис. 4. Структура $180^{\circ}$-ДГ для значений параметров $\mathfrak{x}_{1}=12, \mathfrak{x}_{2}=2, Q=5, K_{u}>0$, представленная в виде графиков зависимостей аналогично рис. 3 .

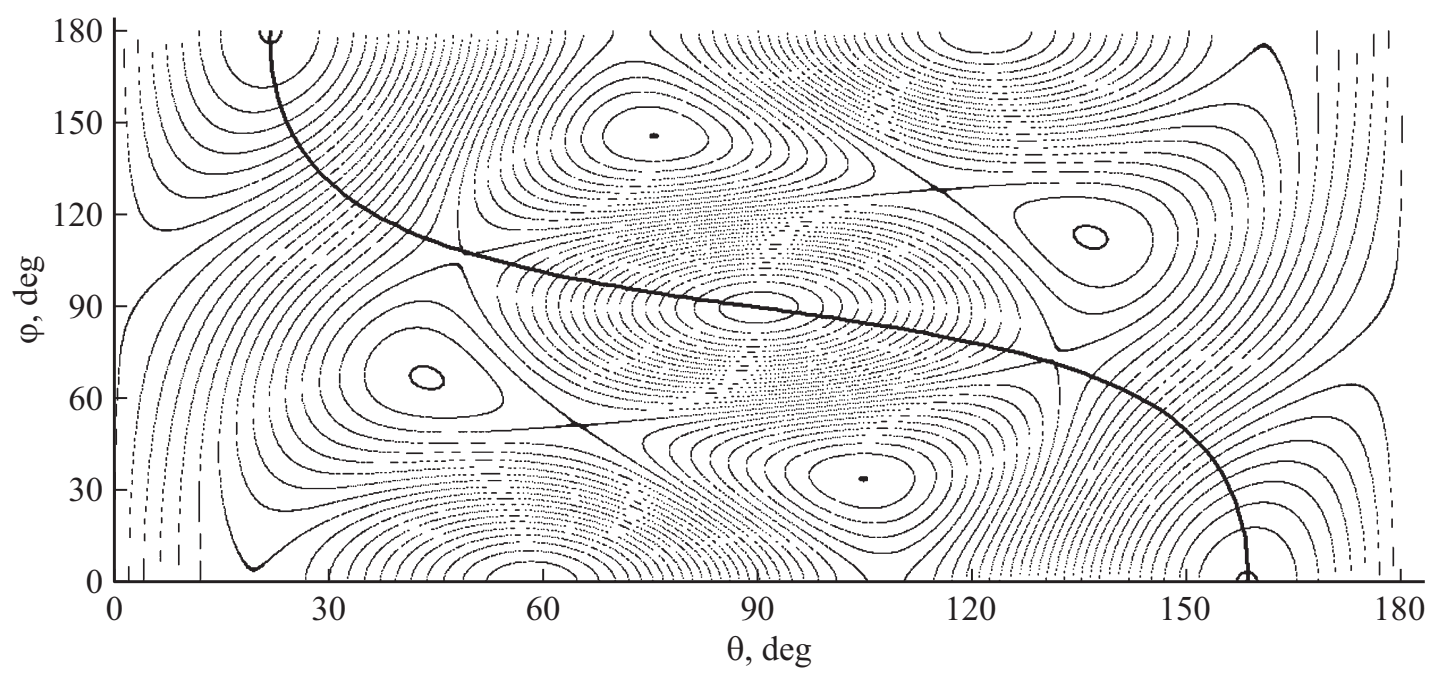

Рис. 5. Изолинии функции $f(\theta, \varphi)$, на котором изображена траектория вектора намагниченности т; ей соответствует структура $180^{\circ}$-ДГ, представленная на рис. 4.

релаксации [18]. Полученные таким образом численные решения сопоставлялись с траекторией вектора намагниченности m на поверхности единичной сферы, наложенной на изолинии функции $f(\theta, \varphi)$. Последняя представляет собой приведенную к величине $K_{u}[15,17]$ часть плотности энергии изучаемого магнетика.

Рассмотрим вначале область значений $K_{u}>0$, которой соответствует ОФД на рис. $1(a, b)$. Для анализа воз- 


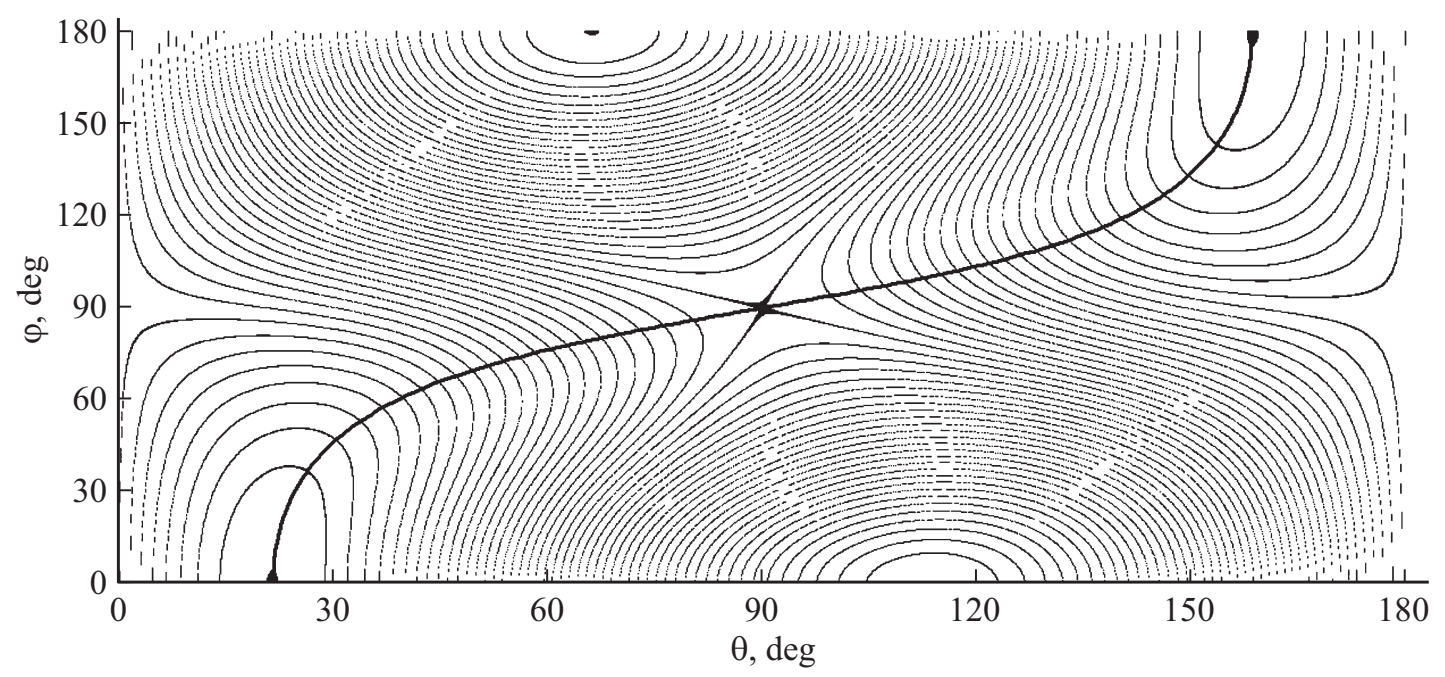

Рис. 6. Изолинии функции $f(\theta, \varphi)$ для значений параметров $\mathfrak{x}_{1}=-1, \mathfrak{x}_{2}=2, Q=5, K_{u}>0$ (аналогично рис. 5) и траектория вектора намагниченности $\mathbf{m}$.

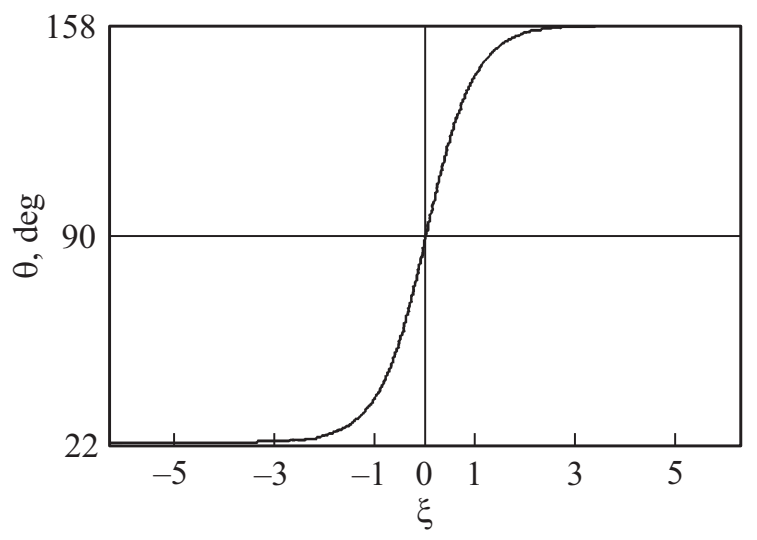

$a$

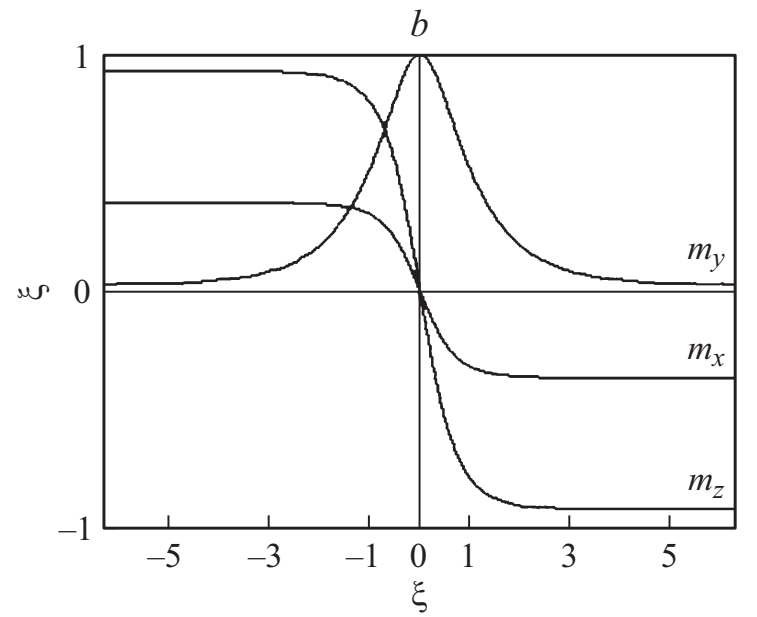

Рис. 7. Структура $180^{\circ}$-ДГ для значений параметров $\mathfrak{x}_{1}=-4, \mathfrak{x}_{2}=2, Q=5, K_{u}>0$, представленная аналогично рис. 3 .

можных типов ДГ в исследуемом случае удобно выбрать значения параметров $\mathfrak{x}_{1}, \mathfrak{x}_{2}$, таким образом, чтобы они на ОФД лежали на некоторой (заранее выбранной) линии (термодинамический путь). Для определенности термодинамический путь выберем вдоль линии $\mathfrak{x}_{2}=2$.
Тогда для значений параметров $\mathfrak{1}_{1}=-2, \mathfrak{x}_{2}=2$, при которых устойчива угловая фаза $P_{[u v 0]}$, решение уравнений (6) будет соответствовать $180^{\circ}$-ДГ с неблоховским распределением намагниченности М (рис. 3), т. е. с выходом намагниченности из плоскости вращения магнитных 
$a$
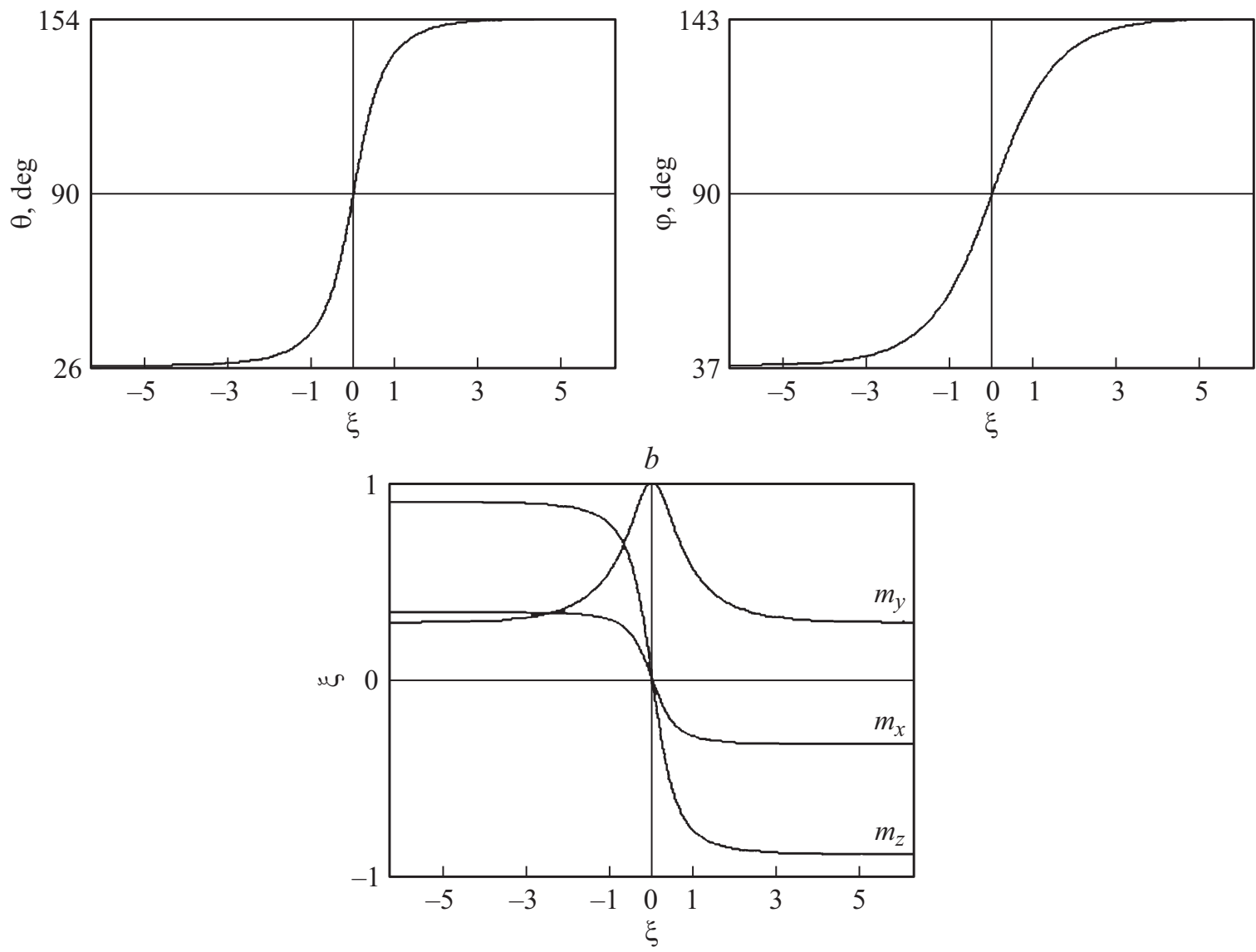

Рис. 8. Структура $180^{\circ}$-ДГ при значении параметров $\mathfrak{x}_{1}=4 \mathfrak{x}_{2}=-1.1, Q=5, K_{u}<0$.

моментов $(\operatorname{div} \mathbf{M}=0)$. Если рассмотреть точку на этой линии с координатами $\mathfrak{x}_{1}=12, \mathfrak{x}_{2}=2$, то для данных значений параметров будет иметь место квазиблоховская $180^{\circ}$-ДГ с перетяжкой (рис. 4). Здесь под перетяжкой понимается участок в профиле ДГ, на котором зависимости $\theta=\theta(y)$ и $\varphi=\varphi(y)$ имеют три точки перегиба [19]. Такая ситуация возможна, если в плоскости вращения магнитных моментов имеется направление, которому соответствует метастабильная магнитная фаза. В данном случае такой метастабильной фазой является $P_{[001]}$; вблизи оси [001] происходит задержка вращения магнитных моментов, что приводит к образованию перетяжки. Как показывают исследования $[7,19]$, перетяжка является зародышем новой фазы (стеночный механизм зародышеобразования). При приближении магнетика к точке СПФП первого рода перетяжка неограниченно разрастается, способствуя безгистерезисному СПФП магнетика из одного состояния в другое. На рис. 5, видно, что на картине изолиний точке с $\theta=\pi / 2, \varphi=\pi / 2$ соответствует особая точка типа „центр“ [20]. Если двигаться на ОФД вдоль выбранного термодинамического пути, то при значениях параметров $\mathfrak{x}_{1}=-1, \mathfrak{x}_{2}=2$ точке $\theta=\pi / 2, \varphi=\pi / 2$ будет соответствовать особая точка типа „седло“ (рис. 6). Анализ показывает, что в этом случае только на графике зависимости $\varphi=\varphi(\xi)$ будет иметь место перетяжка, а на графике $\theta=\theta(y)-$ нет. В данной ситуации область ориентаций магнитных моментов вблизи направления М с $\theta=\pi / 2, \varphi=\pi / 2$ уже не будет являться зародышем новой фазы.

Если на ОФД (рис. $1, a, b)$ двигаться дальше вдоль термодинамического пути $æ_{2}=2$ в сторону отрицательных значений параметра $æ_{1}$, то можно попасть в область, ограниченную линиями $5-N_{1}$ и $7-N_{1}$, с точкой их пересечения в $N_{1}$. Здесь устойчива фаза общего вида $P_{[u v w]}^{\mathrm{I}}$; доменная стенка, соответствующая этой фазе для значений $\mathfrak{1}_{1}=-4, \mathfrak{x}_{2}=2$ также будет являться $180^{\circ}$-ДГ с некруговой траекторией вектора намагниченности (рис. 7).

При $K_{u}<0$ качественная картина микромагнитных структур, возможных в этом случае, существенно не меняется. В частности, это наглядно видно на примере, рассмотренном ранее, когда изменения состояния магнетика можно рассматривать вдоль термодинамического пути, определяемого линией $\mathfrak{x}_{1}=4$ (пунктирная линия на рис. 2). В данном случае магнетик претерпевает ряд магнитных превращений, попеременно находясь в состояниях, соответствующих то угловой фазе (фазы $P_{[u v 0]}^{\mathrm{II}}$ и $\left.P_{[u v 0]}^{\mathrm{I}}\right)$, то симметричной фазе $P_{[001]}$, то опять угловой фазе $\left(P_{[u v 0]}^{\mathrm{III}}\right)$. Анализ системы уравнений (6) 


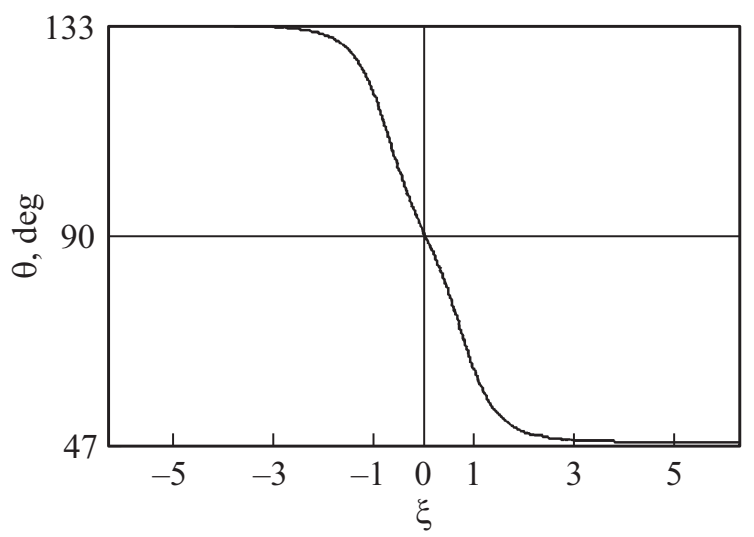

$a$
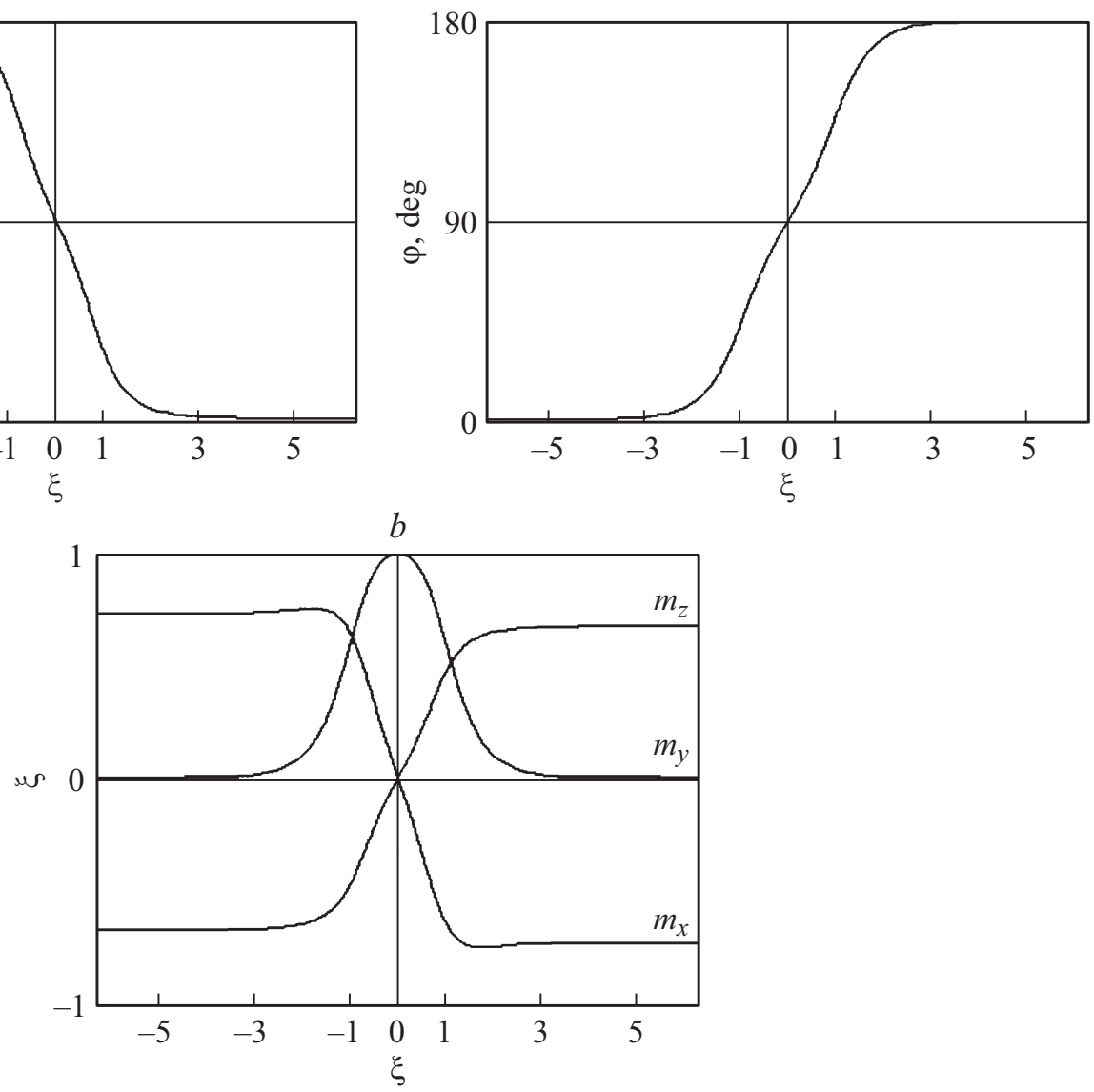

Pис. 9. Структура $180^{\circ}$-ДГ при значениях параметров $\mathfrak{x}_{1}=4, \mathfrak{x}_{2}=1, Q=5, K_{u}<0$.

показывает, что в области значений параметров, соответствующих угловым фазам $P_{[u v 0]}^{\mathrm{II}}, P_{[u v 0]}^{\mathrm{I}}, P_{[u v 0]}^{\mathrm{III}}$ (рис. 2), имеют место решения, отвечающие $180^{\circ}$-ДГ с некруговой траекторией вектора намагниченности М. Характерной особенностью данных неоднородностей является наличие перетяжек в их структуре (как и в случае $K_{u}>0$, рис. 4), что обусловлено наличием в плоскости вращения магнитных моментов оси [001], которая в областях на ОФД (рис. 2), ограниченных прямыми $1-N_{4}$ и $2-N_{4}$ (с точкой их пересечения в $N_{4}$ ), а также прямыми $8-N_{3}$ и $11-N_{4}$ (с точкой их пересечения в начале координат), соответствует метастабильной фазе. В случае, когда термодинамический путь, пересекая линию $2-N_{4}$, уходит в область выше нее, или когда он уходит ниже линии $8-N_{3}$ (т.е. выходит за пределы области существования симметричной фазы $\left.P_{[001]}\right)$, то данная перетяжка в структуре $180^{\circ}$-ДГ исчезает. Следует отметить, что в области на ОФД, лежащей между линиями 9 и 10 (с точкой их пересечения в $C_{3}$ ), фаза $P_{[u v 0]}^{\mathrm{I}}$ является также метастабильной, однако соответствующая ей ось не лежит в плоскости вращения спинов в $180^{\circ}$-ДГ (рис. 8). Поэтому последняя не содержит перетяжки, отвечающей этой оси, а имеет лишь одну перетяжку, отвечающую оси [001].
В области, ограниченной прямыми $1-N_{4}, 8-N_{3}$ и кривой $N_{3} N_{4}$, является устойчивой симметричная фаза $P_{[001]}$. Для значений параметров $\mathfrak{x}_{1}=4, \mathfrak{x}_{2}=1$, соответствующих тоже в этой области, решение уравнений (6) описывает магнитную неоднородность, представленную на рис. 9. В данном случае она соответствуют $180^{\circ}$-ДГ с некруговой траекторией намагниченности. Ее структура такова, что относительно функции $\theta=\theta(y)$ eе можно отнести к $0^{\circ}$-ДГ, а относительно $\varphi=\varphi(y)-$ к $180^{\circ}$-ДГ. Учитывая, что направления $\mathbf{M}$ в соседних доменах противоположны $\left(\mathbf{M}_{1}\left\|[001], \mathbf{M}_{2}\right\|[00 \overline{1}]\right)$, то ДГ в общем случае является 180-градусной. Дальнейший анализ системы уравнений, проведенный в соответствии с данными ОФД, не дает новых решений, качественно отличающихся от полученных ранее. Это позволяет утверждать, что найденные типы магнитных неоднородностей дают полную картину микромагнитных структур, возможных в рассматриваемом магнетике.

\section{4. Выводы}

Таким образом, проведенные исследования показывают, что (210)-ориентированная пластина ферритов- 
гранатов является достаточно сложным объектом для изучения ее магнитных состояний, что обусловлено низкой симметрией магнитной системы. В ней возможны три вида магнитных фаз, различающихся трансформационными свойствами: симметричная $\left(P_{[001]}\right)$, угловая $\left(P_{[u v 0]}\right)$ и пространственная $\left(P_{[u v w]}\right)$ фазы. Между первыми двумя фазами могут иметь место только СПФП первого рода, а между двумя последними фазами СПФП как первого, так и второго рода. Кроме того, в области существования низкосимметричных фаз возможны изоструктурные СПФП первого рода. Неоднородные магнитные состояния магнетика также обладают рядом особенностей, однако характерной их чертой является выход намагниченности из плоскости стенки, т.е. ДГ, возможные в пластине (210), всегда имеют неблоховскую структуру. По этому признаку изучаемый магнетик существенно отличается от (011)- и (111)-ориентированных пластин ферритов-гранатов [17,21], в которых $180^{\circ}$-ДГ с некруговой траекторией вектора намагниченности могут существовать не при всех значениях параметров материала, а лишь при определенных их значениях. Последнее, возможно, и является причиной, объясняющей особенности проявления МЭ в ферритгранатовых пленках с разной ориентацией развитой поверхности, обнаруженные в [4].

В заключение авторы выражают признательность доценту кафедры теоретической физики В.В. Плавскому за ценные замечания и советы.

\section{Список литературы}

[1] В.В. Рандошкин, В.И. Чани, М.В. Логунов, Ю.А. Сажин, В.П. Клин, Б.П. Нам, А.Г. Соловьев, А.Я. Червоненкис. Письма в ЖТФ 15, 42 (1989).

[2] I. Nistor, C. Holthaus, S. Tkachuk. J. Appl. Phys. 101, 09C526 (2007).

[3] Б.Б. Кричевцов, В.В. Павлов, Р.В. Писарев. Письма в ЖЭТФ 49, 466 (1989).

[4] A.S. Logginov, G.A. Meshkov, A.V. Nikolaev, E.P. Nikolaeva, A.P. Pyatakov. Appl. Phys. Lett. 93, 182510 (2008).

[5] А.П. Пятаков, А.С. Сергеев, Е.П. Николаева, Т.Б. Косых, А.В. Николаев, К.А. Звездин, А.К. Звездин. УФН 185, 1077 (2015).

[6] А.Ф. Кабыченков, Ф.В. Лисовский, Е.Г. Мансветова. Письма в ЖЭТФ 97, 304 (2013).

[7] К.П. Белов, А.К. Звездин, А.М. Кадомцева, Р.З. Левитин. Ориентационные переходы в редкоземельных магнетиках. Наука, М. (1979). 320 c.

[8] S. Tkachuk, D. Bowen, C. Krafft, I.D. Mayergoyz. J. Appl. Phys. 105, 07A524 (2009).

[9] Г.В. Арзамасцева, А.М. Балбашов, Ф.В. Лисовский, Е.Г. Мансветова, А.Г. Темирязев, М.П. Темирязева. ЖЭТФ 147, 793 (2015).

[10] В.А. Бородин, В.Д. Дорошев, Т.Н. Тарасенко. ФТТ 27, 583 (1985).

[11] Ю.М. Гуфан. Структурные фазовые переходы. Наука, М. (1982). 304 c.
[12] В.Д. Бучельников, С.В. Таскаев, В.С. Романов, Р.М. Вахитов. ФММ 94, 14 (2002).

[13] Л.Д. Ландау, И.М. Лифшиц. Статистическая физика. Ч. 1. Наука, М. (1995). 608 с.

[14] Р.М. Вахитов. ФММ 89, 16 (2000).

[15] A. Hubert, R. Schafer. Magnetic domains. Springer-Verlag, Berlin (2007). 696 p.

[16] Е.Б. Магадеев, Р.М. Вахитов. ТМФ 171, 511 (2012).

[17] В.В. Плавский, М.А. Шамсутдинов, Б.Н. Филиппов. ФММ 88, 22 (1999).

[18] А.А. Самарский, А.В. Гулин. Численные методы. Наука, M. (1989). 432 c.

[19] Р.М. Вахитов, А.Р. Юмагузин. ФТТ 43, 65 (2001).

[20] Н.В. Карлов, Н.А. Кириченко. Колебания, волны, структуры. Физматлит, М. (2001). 496 с.

[21] Р.М. Вахитов, Е.Г. Шанина. ЖТФ 73, 67 (2003). 